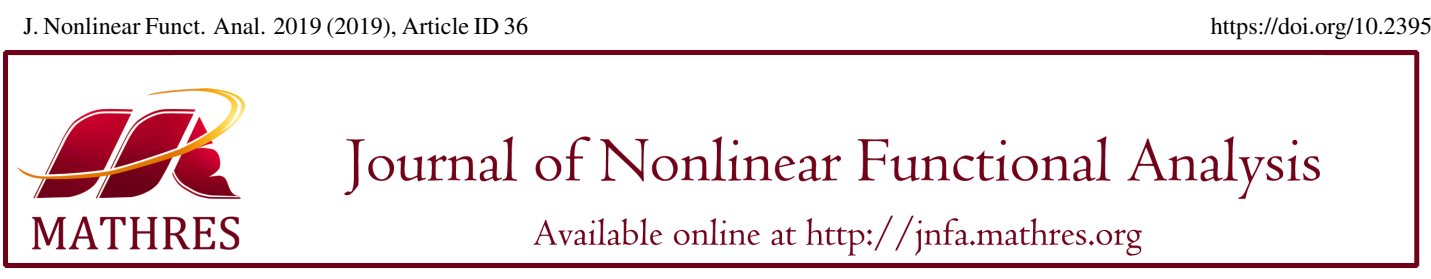

https://doi.org/10.23952/jnfa.2019.36

\title{
PERSISTENCE AND EXTINCTION OF A STOCHASTIC DELAY COMPETITIVE SYSTEM UNDER REGIME SWITCHING
}

\author{
AHMADJAN MUHAMMADHAJI, AZHAR HALIK* \\ College of Mathematics and Systems Science, Xinjiang University, Urumqi 830046, China
}

\begin{abstract}
A class of two species stochastic delay Lotka-Volterra competitive system under regime switching is proposed and discussed. Some sufficient conditions on the extinction, non-persistence in the mean and weak persistence of the solutions are established. The critical value between weak persistence and extinction is obtained.
\end{abstract}

Keywords. Competitive system; Markov switching; Delay; Persistence; Extinction.

2010 Mathematics Subject Classification. 60H10, 92D30.

\section{INTRODUCTION AND PRELIMINARIES}

As an important class of dynamical systems, Lotka-Volterra systems have been become the most important means to explain the ecological phenomena and widely used in many other scientific fields. Lotka-Volterra competition system is one of the most important topics in the study of population dynamical systems. Recently, a lot of results were obtained on Lotka-Volterra competition systems; see, e.g., $[1,2,3,4,5,6,7,8,9]$ and the references cited therein. The traditional two species nonautonomous Lotka-Volterra system takes the form

$$
\begin{aligned}
& \frac{d x_{1}(t)}{d t}=x_{1}(t)\left[r_{1}(t)-a_{11}(t) x_{1}(t)-a_{12}(t) x_{2}(t)\right] \\
& \frac{d x_{2}(t)}{d t}=x_{2}(t)\left[r_{2}(t)-a_{21}(t) x_{1}(t)-a_{22}(t) x_{2}(t)\right],
\end{aligned}
$$

where $r_{i}(t), a_{i j}(t)(i, j=1,2)$ are functions assumed to be continuous and bounded above and below by positive constants. Recently, many authors studied the properties of system (1.1); see [1, 2, 3, 4]. However, in the real world, the growth rate of a natural species will not often respond immediately to changes in its own population or that of an interacting species but will rather do so after a time lag [5]. Results $[5,6,7,8,9]$ show that time delays have a great destabilizing influence on the species population.

${ }^{*}$ Corresponding author.

E-mail addresses: ahmatjanam@aliyun.com (A. Muhammadhaji), azhar1117@163.com (A. Halik).

Received January 6, 2019; Accepted July 23, 2019.

(C)2019 Journal of Nonlinear Functional Analysis 
Thus, we further consider system (1.1) and take the time delay into the system. System (1.1) becomes the following system

$$
\begin{aligned}
& \frac{d x_{1}(t)}{d t}=x_{1}(t)\left[r_{1}(t)-a_{11}(t) x_{1}(t)-a_{12}(t) x_{2}\left(t-\tau_{1}\right)\right] \\
& \frac{d x_{2}(t)}{d t}=x_{2}(t)\left[r_{2}(t)-a_{21}(t) x_{1}\left(t-\tau_{2}\right)-a_{22}(t) x_{2}(t)\right],
\end{aligned}
$$

where $\tau_{i}>0(i=1,2)$ represents the time delay.

On the other hand, stochastic population dynamical systems are extensively studied in recent years; see, e.g., $[10,11,12,13,14,15,16,17,18,19]$ and the references therein. The important hot spot is how to extend these deterministic systems to the case of stochastic systems, because population dynamics is inevitably affected by the environmental white noise which is an important component in an ecosystem. There are various types of environmental noise. For example, telegraph noise and the growth rates are often affected by the telegraph noise. Moreover, the growth rates of some populations in the dry season will be different from those in the rainy season [12]. Several authors pointed out that we can model telegraph noise by a continuous-time Markov chain $\gamma(t), t \geq 0$ with finite-state space $\mathbb{S}=\{1, \cdots, m\}$; see $[10,11,12,13,14]$. In light of the above analysis, we will consider the stochastic effects for system (1.2).

Let the Markov chain $\gamma(t)$ is generated by $Q=\left(q_{i j}\right)$, that is,

$$
P\{\gamma(t+\Delta t)=j \mid \gamma(t)=i\}=\left\{\begin{array}{l}
q_{i j} \Delta t+o(\Delta t), \text { if } j \neq i \\
1+q_{i j} \Delta+o(\Delta t), \text { if } j=i,
\end{array}\right.
$$

where $q_{i j} \geq 0$ for $i, j=1,2, \cdots, m$ with $j \neq i$ and $\sum_{j=1}^{m} q_{i j}=0$ for $i=1,2, \cdots, m$. Then system (1.2) becomes

$$
\begin{aligned}
& \frac{d x_{1}(t)}{d t}=x_{1}(t)\left[r_{1}(\gamma(t))-a_{11}(\gamma(t)) x_{1}(t)-a_{12}(\gamma(t)) x_{2}\left(t-\tau_{1}\right)\right], \\
& \frac{d x_{2}(t)}{d t}=x_{2}(t)\left[r_{2}(\gamma(t))-a_{21}(\gamma(t)) x_{1}\left(t-\tau_{2}\right)-a_{22}(\gamma(t)) x_{2}(t)\right] .
\end{aligned}
$$

If $\gamma(0)=\kappa \in \mathbb{S}$, then (1.3) obeys

$$
\begin{aligned}
& \frac{d x_{1}(t)}{d t}=x_{1}(t)\left[r_{1}(\kappa)-a_{11}(\kappa) x_{1}(t)-a_{12}(\kappa) x_{2}\left(t-\tau_{1}\right)\right] \\
& \frac{d x_{2}(t)}{d t}=x_{2}(t)\left[r_{2}(\kappa)-a_{21}(\kappa) x_{1}\left(t-\tau_{2}\right)-a_{22}(\kappa) x_{2}(t)\right]
\end{aligned}
$$

for a random amount of time until the Markov chain $\gamma(t)$ jumps to another state, say, $\varsigma \in \mathbb{S}$. Then the model satisfies

$$
\begin{aligned}
& \frac{d x_{1}(t)}{d t}=x_{1}(t)\left[r_{1}(\varsigma)-a_{11}(\varsigma) x_{1}(t)-a_{12}(\varsigma) x_{2}\left(t-\tau_{1}\right)\right], \\
& \frac{d x_{2}(t)}{d t}=x_{2}(t)\left[r_{2}(\varsigma)-a_{21}(\varsigma) x_{1}\left(t-\tau_{2}\right)-a_{22}(\varsigma) x_{2}(t)\right],
\end{aligned}
$$

for a random amount of time until $\gamma(t)$ jumps to a new state again. Now we take a further step by considering the environmental noise, namely, the white noise. Then system (1.2) will change significantly. Let $r_{i}(k)(i=1,2)$ denote the growth rate in regime $k(k \in \mathbb{S})$. We usually estimate it by an error term plus an average value. Frequently, the error term follows a normal distribution. Therefore we can replace $r_{i}(k)$ by $r_{i}(k)+\sigma_{i 1}(k) \dot{B}_{i 1}(t)(i=1,2)$ (see, e.g., $\left.[10,11,12,13]\right)$, where $\dot{B}_{i 1}(t)(i=1,2)$ is a white noise and $\sigma_{i 1}^{2}(k)$ represents the intensity of the white noise. Similarly, $-a_{i i}(k)(i=1,2)$ and $-a_{i j}(k)(i, j=1,2, i \neq j)$ will 
become $-a_{i i}(k)+\sigma_{i 2}(k) \dot{B}_{i 2}(t)(i=1,2)$ and $-a_{i j}(k)+\sigma_{i 3}(k) \dot{B}_{i 3}(t)(i, j=1,2, i \neq j)$ (see, e.g., [12, 15]), respectively. Then we obtain the following stochastic delay Lotka-Volterra competitive system under regime switching

$$
\begin{aligned}
d x_{1}(t)= & x_{1}(t)\left[r_{1}(\gamma(t))-a_{11}(\gamma(t)) x_{1}(t)-a_{12}(\gamma(t)) x_{2}\left(t-\tau_{1}\right)\right] d t+\sigma_{11}(\gamma(t)) x_{1}(t) d B_{11}(t) \\
& +\sigma_{12}(\gamma(t)) x_{1}^{2}(t) d B_{12}(t)+\sigma_{13}(\gamma(t)) x_{1}(t) x_{2}\left(t-\tau_{1}\right) d B_{13}(t), \\
d x_{2}(t)= & x_{2}(t)\left[r_{2}(\gamma(t))-a_{21}(\gamma(t)) x_{1}\left(t-\tau_{2}\right)-a_{22}(\gamma(t)) x_{2}(t)\right] d t+\sigma_{21}(\gamma(t)) x_{2}(t) d B_{21}(t) \\
& +\sigma_{22}(\gamma(t)) x_{2}^{2}(t) d B_{22}(t)+\sigma_{23}(\gamma(t)) x_{2}(t) x_{1}\left(t-\tau_{2}\right) d B_{23}(t),
\end{aligned}
$$

where $B_{i}(t)=\left(B_{i 1}(t), B_{i 2}(t), B_{i 3}(t)\right)(i=1,2)$ is a standard white noise, namely, $B_{i}(t)$ is a Brownian motion defined on a complete probability space $\left(\Omega, \digamma,\left\{\digamma_{t}\right\}_{t \geq 0}, P\right)$. Assume that the Markov chain $\gamma(\cdot)$ is independent of $B_{i}(t)$ and has a unique stationary distribution $\pi=\left(\pi_{1}, \pi_{2}, \cdots, \pi_{m}\right)$ which can be obtained by solving the following linear equation $\pi Q=0$ subject to $\sum_{k=1}^{m} \pi_{k}=1$ and $\pi_{k}>0, k \in \mathbb{S}$. In this paper, we always assume that $\min _{k \in \mathbb{S}} a_{i i}(k)>0(i=1,2), \min _{k \in \mathbb{S}} a_{i j}(k) \geq 0(i, j=1,2, i \neq j)$ and $\min _{k \in \mathbb{S}} \sigma_{i 2}^{2}(k)>0$ $(i=1,2), k \in \mathbb{S}$. Next, we define the following notations for the sake of convenience

$$
\hat{f}=\max _{k \in \mathbb{S}} f(k), \check{f}=\inf _{k \in \mathbb{S}} f(k) .
$$

\section{MAIN RESULTS}

In this section, we present the main results of this paper.

Theorem 2.1. (1.4) has a unique and positive solution on $t \geq-\tau$ a.s. (almost surely) for any initial data $\left\{\left(x_{1}(t), x_{2}(t)\right):-\tau \leq t \leq 0\right\} \in C\left([-\tau, 0], R_{+}^{2}\right)$ and $\gamma(0)$, where $\mathbb{R}_{+}^{2}=\left\{x \in \mathbb{R}^{2}: x_{i}>0, i=1,2\right\}$ and $\tau=\max \left\{\tau_{1}, \tau_{2}\right\}$

Proof. Note that the coefficients of the equation are locally Lipschitz continuous. For any given initial value $x_{0} \in R_{+}^{2}$, there is a unique maximal local solution $x(t)=\left(x_{1}(t), x_{2}(t)\right)$ on $t \in\left(-\infty, \tau_{e}\right]$, the explosion time. To show that this solution is global, we need to show that $\tau_{e}=\infty$ a.s. Let $k_{0}>0$ be sufficiently large for every component of $x_{0}$ lying within interval $\left[1 / k_{0}, k_{0}\right]$. For each integer $k \geq k_{0}$, define the stopping time

$$
\tau_{k}=\inf \left\{t \in\left[0, \tau_{e}\right): x_{i}(t) \notin(1 / k, k) \text { for some } i=1,2 .\right\}
$$

with usual setting $\inf \emptyset$, where $\emptyset$ denotes the empty set. Clearly, $\tau_{k}$ is increasing as $k \rightarrow \infty$. Set $\tau_{\infty}=$ $\lim _{k \rightarrow \infty} \tau_{k}$, whence $\tau_{\infty} \leq \tau_{e}$ a.s. If we can prove $\tau_{\infty}=\infty$ a.s., then $\tau_{e}=\infty$ a.s., which implies the desired result. To prove this statement, let us define a $C^{2}$-function $V: R_{+}^{2} \rightarrow R_{+}$by

$$
V(x(t))=\sum_{i=1}^{2} u\left(x_{i}\right),
$$

where $x(t)=\left(x_{1}(t), x_{2}(t)\right), u\left(x_{i}\right)=\ln x_{i}$. Clearly, $u(\cdot) \geq 0$ and $u\left(0^{+}\right)=u(\infty)=\infty$. Let $T>0$ be arbitrary. For $0 \leq t \leq \tau_{k} \wedge T$, applying the Ito formula (see [17]) to $V(x(t))$, we obtain that

$$
D V(x(t))=L V(x(t)) d t+M(t),
$$


where

$$
\begin{aligned}
L V(x(t))= & {\left[r_{1}-a_{11} x_{1}(t)-a_{12} x_{2}\left(t-\tau_{1}\right)-0.5 \sigma_{11}^{2}-0.5 \sigma_{12}^{2} x_{1}^{2}-0.5 \sigma_{13}^{2} x_{2}^{2}\left(t-\tau_{1}\right)\right.} \\
& \left.+r_{2}-a_{21} x_{1}\left(t-\tau_{2}\right)-a_{22} x_{2}(t)-0.5 \sigma_{21}^{2}-0.5 \sigma_{22}^{2} x_{2}^{2}-0.5 \sigma_{23}^{2} x_{1}^{2}\left(t-\tau_{2}\right)\right] d t,
\end{aligned}
$$

and

$$
\begin{aligned}
M(t)= & \sigma_{11} d B_{11}+\sigma_{12} x_{1}(t) d B_{12}+\sigma_{13} x_{2}\left(t-\tau_{1}\right) d B_{13} \\
& +\sigma_{21} d B_{21}+\sigma_{22} x_{2}(t) d B_{22}+\sigma_{23} x_{1}\left(t-\tau_{2}\right) d B_{23},
\end{aligned}
$$

where we drop $t$ from $r_{i}(t), a_{i j}(t), \sigma_{i j}(t), B_{i j}(t)$. From (2.1) we have

$$
\begin{aligned}
& r_{1}-a_{11} x_{1}(t)-a_{12} x_{2}\left(t-\tau_{1}\right)-0.5 \sigma_{11}^{2}-0.5 \sigma_{12}^{2} x_{1}^{2}-0.5 \sigma_{13}^{2} x_{2}^{2}\left(t-\tau_{1}\right) \\
& +r_{2}-a_{21} x_{1}\left(t-\tau_{2}\right)-a_{22} x_{2}(t)-0.5 \sigma_{21}^{2}-0.5 \sigma_{22}^{2} x_{2}^{2}-0.5 \sigma_{23}^{2} x_{1}^{2}\left(t-\tau_{2}\right),
\end{aligned}
$$

which is bounded, say by $K_{1}$, in $R_{+}$. We therefore obtain

$$
d V(x(t)) \leq K_{1} d t+M(t),
$$

as long as $x(t) \in R_{+}^{2}$. Integrating both sides from 0 to $\tau_{k} \wedge T$, and taking expectations yield

$$
\mathbb{E} V\left(x\left(\tau_{k} \wedge T\right)\right) \leq V\left(x_{0}\right)+K_{1} \mathbb{E}\left(\tau_{k} \wedge T\right) \leq V\left(x_{0}\right)+K_{1} T:=K_{T} .
$$

By the definition of $\tau_{k}, x_{i}\left(\tau_{k}\right)=k$ or $1 / k$ for some $i=1,2$,

$$
\begin{aligned}
\mathbb{P}\left(\tau_{k} \leq T\right)\left[u\left(k^{-1}\right) \wedge u(k)\right] & \leq \mathbb{P}\left(\tau_{k} \leq T\right) V\left(x\left(\tau_{k} \leq T\right)\right) \\
& \leq \mathbb{E} V\left(x\left(\tau_{k} \leq T\right)\right) \\
& \leq K_{T}
\end{aligned}
$$

which implies that

$$
\lim _{k \rightarrow \infty} \sup \mathbb{P}\left(\tau_{k} \leq T\right) \leq \lim _{k \rightarrow \infty} \frac{K_{T}}{u\left(k^{-1}\right) \wedge u(k)}=0 .
$$

Since $T>0$ is arbitrary, we have $\mathbb{P}\left(\tau_{\infty}<\infty\right)=0$ as required. This completes the proof.

Theorem 2.2. If $\sum_{i=1}^{m} \pi_{i} b_{1}(i)<0$ and $\sum_{i=1}^{m} \pi_{i} b_{2}(i)<0$, then the population $x_{i}(t)$ represented by model (1.4) goes to extinction a.s. (almost surely), i.e., $\lim _{t \rightarrow+\infty} x_{i}(t)=0$, where $b_{i}(\gamma)=r_{i}(\gamma)-0.5 \sigma_{i 1}^{2}(\gamma)$.

Proof. Applying the Itô formula (see [17]) to the first equation of system (1.4) gives

$$
\begin{aligned}
d \ln x_{1}(t)= & {\left[r_{1}(\gamma(t))-a_{11}(\gamma(t)) x_{1}(t)-a_{12}(\gamma(t)) x_{2}\left(t-\tau_{1}\right)-0.5 \sigma_{11}^{2}(\gamma(t))\right.} \\
& \left.-0.5 \sigma_{12}^{2}(\gamma(t)) x_{1}^{2}-0.5 \sigma_{13}^{2}(\gamma(t)) x_{2}^{2}\left(t-\tau_{1}\right)\right] d t+\sigma_{11}(\gamma(t)) d B_{11}(t) \\
& +\sigma_{12}(\gamma(t)) x_{1}(t) d B_{12}(t)+\sigma_{13}(\gamma(t)) x_{2}\left(t-\tau_{1}\right) d B_{13}(t) .
\end{aligned}
$$

This further implies that

$$
\begin{aligned}
\ln x_{1}(t)-\ln x_{1}(0)= & \int_{0}^{t}\left[b_{1}(\gamma(s))-a_{11}(\gamma(s)) x_{1}(s)-a_{12}(\gamma(s)) x_{2}\left(s-\tau_{1}\right)\right. \\
& \left.-0.5 \sigma_{12}^{2}(\gamma(s)) x_{1}^{2}(s)-0.5 \sigma_{13}^{2}(\gamma(s)) x_{2}^{2}\left(s-\tau_{1}\right)\right] d s+\sum_{i=1}^{3} M_{i}(t),
\end{aligned}
$$

where

$$
\begin{gathered}
b_{1}(\gamma(s))=r_{1}(\gamma(s))-0.5 \sigma_{11}^{2}(\gamma(s)), \quad M_{1}(t)=\int_{0}^{t} \sigma_{11}(\gamma(s)) d B_{11}(s), \\
M_{2}(t)=\int_{0}^{t} \sigma_{12}(\gamma(s)) x_{1}(s) d B_{12}(s), \quad M_{3}(t)=\int_{0}^{t} \sigma_{13}(\gamma(s)) x_{2}\left(s-\tau_{1}\right) d B_{13}(s) .
\end{gathered}
$$


Note that $M_{1}(t)$ is a local martingale, whose quadratic variation is

$$
<M_{1}(t), M_{1}(t)>=\int_{0}^{t} \sigma_{11}^{2}(\gamma(s)) d(s) \leq \hat{\sigma}^{2}{ }_{1} t .
$$

Making use of the strong law of large numbers for local martingales (see, e.g., [12, 14]) yields

$$
\lim _{t \rightarrow+\infty} M_{1}(t) / t=0 \quad \text { a.s. }
$$

On the other hand,

$$
<M_{2}(t), M_{2}(t)>=\int_{0}^{t} \sigma_{12}^{2}(\gamma(s)) x_{1}^{2}(s) d(s),<M_{3}(t), M_{3}(t)>=\int_{0}^{t} \sigma_{13}^{2}(\gamma(s)) x_{2}^{2}\left(s-\tau_{1}\right) d(s) .
$$

In view of the exponential martingales inequality, for any positive constants $T, \alpha$ and $\beta$, we have

$$
\mathbb{P}\left\{\sup _{0 \leq t \leq T}\left[M_{i}(t)-\frac{\alpha}{2}<M_{i}(t), M_{i}(t)>\right]>\beta\right\} \leq e^{-\alpha \beta}, \quad i=2,3 .
$$

Letting $T=k, \alpha=1$ and $\beta=2 \ln k$, we have

$$
\mathbb{P}\left\{\sup _{0 \leq t \leq k}\left[M_{i}(t)-\frac{1}{2}<M_{i}(t), M_{i}(t)>\right]>2 \ln k\right\} \leq \frac{1}{k^{2}}, \quad i=2,3 .
$$

Using the Borel-Cantelli Lemma (see, e.g., $[12,14]$ ) leads to the fact that, for almost all $\omega \in \Omega$, there is a random integer $k_{0}=k_{0}(\omega)$ such that, for $k \geq k_{0}$, $\sup _{0 \leq t \leq k}\left[M_{i}(t)-\frac{1}{2}<M_{i}(t), M_{i}(t)>\right] \leq 2 \ln k$. Then

$$
\begin{gathered}
M_{2}(t) \leq 2 \ln k+\frac{1}{2}<M_{2}(t), M_{2}(t)>=2 \ln k+0.5 \int_{0}^{t} \sigma_{12}^{2}(\gamma(s)) x_{1}^{2}(s) d s, \\
M_{3}(t) \leq 2 \ln k+\frac{1}{2}<M_{3}(t), M_{3}(t)>=2 \ln k+0.5 \int_{0}^{t} \sigma_{13}^{2}(\gamma(s)) x_{2}^{2}\left(s-\tau_{1}\right) d s,
\end{gathered}
$$

for all $0 \leq t \leq k, k \geq k_{0}$ a.s. Substituting these inequalities into (2.2) gives

$$
\begin{aligned}
\ln x_{1}(t)-\ln x_{1}(0)= & \int_{0}^{t} b_{1}(\gamma(s)) d s-\int_{0}^{t} a_{11}(\gamma(s)) x_{1}(s) d s-\int_{0}^{t} a_{12}(\gamma(s)) x_{2}\left(s-\tau_{1}\right) d s \\
& +M_{1}(t)+4 \ln k \\
\leq & \int_{0}^{t} b_{1}(\gamma(s)) d s+M_{1}(t)+4 \ln k
\end{aligned}
$$

for all $0 \leq t \leq k, k \geq k_{0}$ almost surely. In other words, we have shown that, for $0 \leq k-1 \leq t \leq k$,

$$
t^{-t}\left[\ln x_{1}(t)-\ln x_{1}(0)\right] \leq t^{-t} \int_{0}^{t} b_{1}(\gamma(s)) d s+\frac{4 \ln k}{t}+\frac{M_{1}(t)}{t} \leq t^{-t} \int_{0}^{t} b_{1}(\gamma(s)) d s+\frac{4 \ln k}{k-1}+\frac{M_{1}(t)}{t} .
$$

Making use of (2.3) and the ergodicity of $\gamma(\cdot)$, we have

$$
\limsup _{t \rightarrow+\infty} t^{-1} \ln x_{1}(t) \leq \limsup _{t \rightarrow+\infty} t^{-1} \int_{0}^{t} b_{1}(\gamma(s)) d s=\sum_{i=1}^{m} \pi_{i} b_{1}(i) .
$$

That is to say, if $\sum_{i=1}^{m} \pi_{i} b_{1}(i)<0$, then $\lim _{t \rightarrow+\infty} x_{1}(t)=0$. As for $x_{2}(t)$, the proof is similar to the above discussion. Finally, we have $\sum_{i=1}^{m} \pi_{i} b_{2}(i)<0$. Hence, $\lim _{t \rightarrow+\infty} x_{2}(t)=0$. This completes the proof.

Theorem 2.3. If $\sum_{i=1}^{m} \pi_{i} b_{1}(i)=0$ and $\sum_{i=1}^{m} \pi_{i} b_{2}(i)=0$, then the population is nonpersistent in the mean a.s., i.e., $\lim _{t \rightarrow+\infty} t^{-1} \int_{0}^{t} x_{i}(s) d s=0$, a.s. 
Proof. For given $\varepsilon>0$, there exists a constant $T_{1}=T_{1}(\varepsilon)$ such that

$$
t^{-t} \int_{0}^{t} b_{1}(\gamma(s)) d s \leq \limsup _{t \rightarrow+\infty} t^{-1} \int_{0}^{t} b_{1}(\gamma(s)) d s+\varepsilon / 2=\sum_{i=1}^{m} \pi_{i} b_{1}(i)+\varepsilon / 2=\varepsilon / 2, \quad t \geq T_{1} .
$$

Substituting this inequality into (2.5), one see that

$$
\begin{aligned}
\ln x_{1}(t)-\ln x_{1}(0) & \leq \int_{0}^{t} b_{1}(\gamma(s)) d s-\int_{0}^{t} a_{11}(\gamma(s)) x_{1}(s) d s+M_{1}(t)+4 \ln k \\
& \leq \varepsilon t / 2-\check{a}_{11} \int_{0}^{t} x_{1}(s) d s+M_{1}(t)+4 \ln k,
\end{aligned}
$$

for all $T_{1} \leq t \leq k, k \geq k_{0}$ almost surely. Note that there exists a $T>T_{1}$ such that for all $T \leq k-1 \leq$ $t \leq k$ and $k \leq k_{0}$. We have $(4 \ln k) / t \leq \varepsilon / 4$ and $M_{1} / t \leq \varepsilon / 4$. In other words, we have proved that $\ln x_{1}(t)-\ln x_{1}(0) \leq \varepsilon t-\breve{a}_{11} \int_{0}^{t} x_{1}(s) d s$ for sufficiently large $t>T$. Letting $g(t)=\int_{0}^{t} x_{1}(s) d s$, we obtain

$$
\ln (d g / d t)<\varepsilon t-\check{a}_{11} g(t)+\ln x_{1}(0), \quad t>T .
$$

That is to say $\check{a}_{11}^{-1}\left[e^{\check{a}_{11} g(t)}-e^{\check{a}_{11} g(T)}\right]<x_{1}(0) \varepsilon^{-1}\left[e^{\varepsilon t}-e^{\varepsilon T}\right]$. Rewriting this inequality, we get

$$
e^{\check{a}_{11} g(t)}<e^{\check{a}_{11} g(T)}+x_{1}(0) \check{a}_{11} \varepsilon^{-1} e^{\varepsilon t}-x_{1}(0) \check{a}_{11} \varepsilon^{-1} e^{\varepsilon T} .
$$

Taking logarithm on the both sides leads to

$$
g(t)<\check{a}_{11}^{-1} \ln \left\{x_{1}(0) \check{a}_{11} \varepsilon^{-1} e^{\varepsilon t}+e^{\check{a}_{11} g(T)}-x_{1}(0) \check{a}_{11} \varepsilon^{-1} e^{\varepsilon T}\right\},
$$

that is,

$$
\limsup _{t \rightarrow+\infty} t^{-1} \int_{0}^{t} x_{1}(s) d s \leq \check{a}_{11}^{-1} \limsup _{t \rightarrow+\infty} t^{-1} \ln \left[x_{1}(0) \check{a}_{11} \varepsilon^{-1} e^{\varepsilon t}+e^{\check{a}_{11} g(T)}-x_{1}(0) \check{a}_{11} \varepsilon^{-1} e^{\varepsilon T}\right] .
$$

An application of L'Hospital's rule, one derives

$$
\limsup _{t \rightarrow+\infty} t^{-1} \int_{0}^{t} x_{1}(s) d s \leq \limsup _{t \rightarrow+\infty} \check{a}_{11}^{-1} t^{-1} \ln \left[x_{1}(0) \check{a}_{11} \varepsilon^{-1} e^{\varepsilon t}\right]=\varepsilon / \check{a}_{11} .
$$

Since $\varepsilon$ is arbitrary, we get limsup $\sup _{t \rightarrow+\infty} t^{-1} \int_{0}^{t} x_{1}(s) d s \leq 0$. As for $x_{2}(t)$, the proof is similar to the above discussion. Finally, we have $\lim \sup _{t \rightarrow+\infty} t^{-1} \int_{0}^{t} x_{2}(s) d s \leq 0$. This completes the proof.

Theorem 2.4. If $\sum_{i=1}^{m} \pi_{i} b_{1}(i)>0$ and $\sum_{i=1}^{m} \pi_{i} b_{2}(i)>0$, then the population is weakly persistent a.s., i.e., $\limsup \operatorname{sit}_{t \rightarrow+} x_{i}(t)>0$, a.s.

Proof. First, let us show that

$$
\limsup _{t \rightarrow+\infty} t^{-1} \ln x_{1}(t) \leq 0, \quad \text { a.s. }
$$

By applying the Itô formula to Eq. (1.4) results in

$$
\begin{aligned}
d\left(e^{t} \ln x\right)= & e^{t} \ln x_{1} d t+e^{t} d \ln x_{1} \\
= & e^{t}\left[\ln x_{1}+b_{1}(\gamma)-a_{11}(\gamma) x_{1}-a_{12}(\gamma) x_{2}\left(t-\tau_{1}\right)-0.5 \sigma_{12}^{2}(\gamma) x_{1}^{2}\right. \\
& \left.-0.5 \sigma_{13}^{2}(\gamma) x_{2}^{2}\left(t-\tau_{1}\right)\right] d t+e^{t}\left[\sigma_{11} d B_{11}(t)+\sigma_{12} x_{1} d B_{12}(t)+\sigma_{13} x_{2}\left(t-\tau_{1}\right) d B_{13}(t)\right]
\end{aligned}
$$

Thus, we have shown that

$$
\begin{aligned}
\ln x_{1}(t)-\ln x_{1}(0)= & \int_{0}^{t} e^{s}\left[\ln x_{1}+b_{1}(\gamma(s))-a_{11}(\gamma(s)) x_{1}(s)-a_{12}(\gamma(s)) x_{2}\left(s-\tau_{1}\right)\right. \\
& \left.-0.5 \sigma_{12}^{2}(\gamma(s)) x_{1}^{2}(s)-0.5 \sigma_{13}^{2}(\gamma(s)) x_{2}^{2}\left(s-\tau_{1}\right)\right] d s+\sum_{i=1}^{3} N_{i}(t)
\end{aligned}
$$


where

$$
\begin{gathered}
N_{1}(t)=\int_{0}^{t} e^{s} \sigma_{11}(\gamma(s)) d B_{11}(s), \quad N_{2}(t)=\int_{0}^{t} e^{s} \sigma_{12}(\gamma(s)) x_{1}(s) d B_{12}(s), \\
M_{3}(t)=\int_{0}^{t} e^{s} \sigma_{13}(\gamma(s)) x_{2}\left(s-\tau_{1}\right) d B_{13}(s) .
\end{gathered}
$$

The quadratic variations of $N_{1}(t), N_{2}(t)$ and $N_{3}(t)$ are

$$
\begin{aligned}
<N_{1}(t), N_{1}(t)>= & \int_{0}^{t} e^{2 s} \sigma_{11}^{2}(\gamma(s)) d(s),<N_{2}(t), N_{2}(t)>=\int_{0}^{t} e^{2 s} \sigma_{12}^{2}(\gamma(s)) x_{1}^{2}(s) d(s), \\
& <N_{3}(t), N_{3}(t)>=\int_{0}^{t} e^{2 s} \sigma_{13}^{2}(\gamma(s)) x_{2}^{2}\left(s-\tau_{1}\right) d(s) .
\end{aligned}
$$

It follow from (2.4) that (choose $T=\gamma k, \alpha=e^{-\lambda k}, \beta=\theta e^{\lambda k} \ln k$ )

$$
\mathbb{P}\left\{\sup _{0 \leq t \leq \lambda k}\left[N_{i}(t)-0.5 e^{-\lambda k}<N_{i}(t), N_{i}(t)>\right]>\theta e^{\lambda k} \ln k\right\} \leq k^{-\theta}, \quad i=1,2,3 .
$$

where $\theta>1$ and $\lambda>0$ are arbitrary. By virtue of the Borel-Cantelli lemma, for almost all $\omega \in \Omega$, there exists $k_{0}(\omega)$ such that, for every $k \geq k_{0}(\omega)$,

$$
N_{i}(t) \leq 0.5 \exp (-\lambda k)<N_{i}(t), N_{i}(t)>+\theta \exp (\lambda k) \ln k, \quad 0 \leq t \leq \lambda k, \quad i=1,2,3,
$$

that is,

$$
\begin{gathered}
N_{1}(t) \leq 0.5 \exp (-\lambda k) \int_{0}^{t} e^{2 s} \sigma_{11}^{2}(\gamma(s)) d(s)+\theta e^{\lambda k} \ln k, \\
N_{2}(t) \leq 0.5 \exp (-\lambda k) \int_{0}^{t} e^{2 s} \sigma_{12}^{2}(\gamma(s)) x_{1}^{2}(s) d(s)+\theta e^{\lambda k} \ln k, \\
N_{3}(t) \leq 0.5 \exp (-\lambda k) \int_{0}^{t} e^{2 s} \sigma_{13}^{2}(\gamma(s)) x_{2}^{2}\left(s-\tau_{1}\right) d(s)+\theta e^{\lambda k} \ln k,
\end{gathered}
$$

for $0 \leq t \leq \gamma k$. Substituting these inequality into (2.7) yields that

$$
\begin{aligned}
e^{t} \ln x_{1}(t)-\ln x_{1}(0)= & \int_{0}^{t} e^{s}\left[\ln x_{1}+b_{1}(\gamma(s))-a_{11}(\gamma(s)) x_{1}(s)-a_{12}(\gamma(s)) x_{2}\left(s-\tau_{1}\right)\right. \\
& \left.-0.5 \sigma_{12}^{2}(\gamma(s)) x_{1}^{2}(s)-0.5 \sigma_{13}^{2}(\gamma(s)) x_{2}^{2}\left(s-\tau_{1}\right)\right] d s \\
& +0.5 e^{-\lambda k} \int_{0}^{t} e^{2 s} \sigma_{11}^{2}(\gamma(s)) d s+0.5 e^{-\lambda k} \int_{0}^{t} e^{2 s} \sigma_{12}^{2}(\gamma(s)) x_{1}^{2}(s) d s \\
& +0.5 e^{-\lambda k} \int_{0}^{t} e^{2 s} \sigma_{13}^{2}(\gamma(s)) x_{2}^{2}\left(s-\tau_{1}\right) d s+3 \theta e^{\lambda k} \ln k \\
= & \int_{0}^{t} e^{s}\left[\ln x_{1}+b_{1}(\gamma(s))-a_{11}(\gamma(s)) x_{1}(s)-a_{12}(\gamma(s)) x_{2}\left(s-\tau_{1}\right)\right. \\
& \left.+0.5 \sigma_{11}^{2}(\gamma(s)) e^{s-\gamma k}\right] d s-\int_{0}^{t} e^{s} 0.5 \sigma_{12}^{2}(\gamma(s)) x_{1}^{2}(s)\left[1-e^{s-\gamma k}\right] d s \\
& -\int_{0}^{t} e^{s} 0.5 \sigma_{13}^{2}(\gamma(s)) x_{2}^{2}\left(s-\tau_{1}\right)\left[1-e^{s-\gamma k}\right] d s+3 \theta e^{\lambda k} \ln k \\
\leq & \int_{0}^{t} e^{s}\left[\ln x_{1}+\hat{b}_{1}-\check{a}_{11} x_{1}(s)+0.5 \hat{\sigma}_{11}^{2}\right] d s+3 \theta e^{\lambda k} \ln k,
\end{aligned}
$$

where we use the facts that $s \leq \lambda k$ and $a_{12}(\gamma(s)) \geq 0$ in the last inequality. Since $\check{a}_{11}>0$, there exists a constant $C$ independent of k such that $\ln x_{1}+\hat{b}_{1}-\check{a}_{11} x_{1}+0.5 \hat{\sigma}_{11}^{2} \leq C$. In other words,

$$
e^{t} \ln x_{1}(t)-\ln x_{1}(0) \leq C\left[e^{t}-1\right]+3 \theta e^{\lambda k} \ln k, \quad 0 \leq t \leq \lambda k .
$$

If $\lambda(k-1) \leq t \leq \lambda k$ and $k \geq k_{0}(\omega)$, we have $\ln x_{1}(x) / t \leq e^{-t} \ln (0) / t+C\left[1-e^{-t}\right] / t+3 \theta e^{-\lambda(k-1)} e^{\lambda k} \ln k / t$, which becomes the desired assertion (2.6) by setting $t \rightarrow+\infty$. 
Now we suppose $\sum_{i=1}^{m} \pi_{i} b_{1}(i)>0$. We will prove that $\limsup _{t \rightarrow+\infty} x_{1}(t)>0$ a.s. If this assertion is not true, then $\mathbb{P}(F)>0$, where $F=\left\{\limsup _{t \rightarrow+\infty} x_{1}(t)=0\right\}$. It follows from (2.2) that

$$
\begin{aligned}
t^{-1}\left[\ln x_{1}(t)-\ln x_{1}(0)\right]= & t^{-1} \int_{0}^{t} b_{1}(\gamma(s)) d s-t^{-1} \int_{0}^{t} a_{11}(\gamma(s)) x_{1}(s) d s \\
& -t^{-1} \int_{0}^{t} a_{12}(\gamma(s)) x_{2}\left(s-\tau_{1}\right) d s-0.5 t^{-1} \int_{0}^{t} \sigma_{12}^{2}(\gamma(s)) x_{1}^{2}(s) d s \\
& -0.5 t^{-1} \int_{0}^{t} \sigma_{13}^{2}(\gamma(s)) x_{2}^{2}\left(s-\tau_{1}\right) d s+\sum_{i=1}^{3} M_{i}(t) / t .
\end{aligned}
$$

On the other hand, for $\forall \omega \in F$, we have $\limsup _{t \rightarrow+\infty} x_{1}(t, \omega)=0$. Thus it follows from the law of large numbers for local martingales that $\limsup _{t \rightarrow+\infty} M_{i}(t) / t=0, i=1,2,3$. Substituting these inequalities into (2.8) gives $\lim \sup _{t \rightarrow+\infty}\left[\ln x_{1}(t, \omega) / t\right]=\sum_{i=1}^{m} \pi_{i} b_{1}(i)>0$. Then $\mathbb{P}\left(\limsup _{t \rightarrow+\infty}\left[\ln x_{1}(t) / t\right]>0\right)>0$, which leads to a contradiction with (2.6). Then $\lim \sup _{t \rightarrow+\infty} x_{1}(t)>0$. As for $x_{2}(t)$, the proof is similar to the above discussion. Finally, we have

$$
\limsup _{t \rightarrow+\infty} x_{2}(t)>0
$$

This completes the proof.

\section{Discussion}

In this paper, we proposed a stochastic delayed two species non-autonomous competitive system and derived sufficient conditions on the extinction. Non-persistence in the mean and weak persistence were established. The critical value between weak persistence and extinction was obtained.

In [12], the authors considered the following stochastic delay Logistic equation under regime switching

$$
\begin{aligned}
d x(t)= & x(t)[r(\gamma(t))-a(\gamma(t)) x(t)-n(\gamma(t)) x(t-\tau)] d t+\sigma_{1}(\gamma(t)) x(t) d B_{1}(t) \\
& +\sigma_{2}(\gamma(t)) x^{2}(t) d B_{2}(t)+\sigma_{3}(\gamma(t)) x(t) x(t-\tau) d B_{3}(t)
\end{aligned}
$$

where $B(t)=\left(B_{1}(t), B_{2}(t), B_{3}(t)\right)$ denotes a three-dimensional Brownian motion defined on a complete probability space $\left(\Omega, \digamma,\left\{\digamma_{t}\right\}_{t \geq 0}, P\right)$. The critical value between weak persistence and extinction was obtained. In this paper, we generalized the stochastic delay Logistic system (3.1) into the stochastic delayed two species non-autonomous competitive system system (1.4), and we have a same conclusion with [12], that is, the extinction or persistence of $x(t)$ depends only on $\sum_{i=1}^{m} \pi_{i} b(i)$. If $\sum_{i=1}^{m} \pi_{i} b(i)>0$, then the species $x(t)$ is weakly persistent; if $\sum_{i=1}^{m} \pi_{i} b(i)<0$, then the population $x(t)$ goes to extinction. We also obtained that the white noise $\sigma_{1}$ is unfavorable for the persistence of the species, and the white noises $\sigma_{2}$ and $\sigma_{3}$ as well as the time delay $\tau$ have no impact on the extinction and persistence of $x(t)$. However, the Markov chain $\gamma(t)$ has impact on the extinction. The persistence of $x(t)$ and the distribution $\left(\pi_{1}, \cdots, \pi_{m}\right)$ of $\gamma(t)$ play a very important role in determining extinction or persistence of the species.

\section{Funding}

This work was supported by the National Natural Science Foundations of China (Grant Nos. 11601464 and 11662020) . 


\section{REFERENCES}

[1] S. Ahmad, Convergence and ultimate bounds of the nonautonomous Volterra-Lotka competition equation, J. Math. Anal. Appl. 127 (1987), 377-387.

[2] S. Ahmad, On the nonautonomous Volterra-Lotka competition equations, Proc. Amer. Math. Soc. 117 (1993), $199-204$.

[3] Q.L. Peng, L.S. Chen, Asymptotic behavior of the nonautonomous two-species Lotka-Volterra competition models, Comput. Math. Appl. 27 (1994), 53-60.

[4] G.Z. Zeng, L.S. Chen, J.F. Chen, Persistence and periodic orbits for two-species nonautonomous diffusion Lotka-Volterra models, Mathl. Comput. Modelling 20 (1994), 69-80.

[5] A. Muhammadhaji, R. Mahemuti, Z. Teng, Periodic solutions for n-species Lotka-Volterra competitive systems with pure delays, Chinese J. Math. 2015 (2015), Article ID 856959.

[6] Y. Li, Periodic solutions for delay Lotka-Volterra competition systems, J. Math. Anal. Appl. 246 (2000), $230-244$.

[7] A. Muhammadhaji, Z. Teng, L. Fei, Permanence in nonautonomous discrete Lotka-Volterra n-species competitive systems with pure-delays and feedback controls, Int. J. Math. 24 (2013), Article ID 1350053.

[8] Y. Li, K. Zhao, and Y. Ye, Multiple positive periodic solutions of n-species delay competition systems with harvesting terms, Nonlinear Anal. Real World Appl. 12 (2011), 1013-1022.

[9] A. Muhammadhaji, Z. Teng, M, Rehim, On a two species stochastic Lotka-Volterra competition system, J. Dyn. Control. Syst. 21 (2015), 495-511.

[10] Q. Luo, X. Mao, Stochastic population dynamics under regime switching II, J. Math. Anal. Appl. 355 (2009), $577-593$.

[11] X. Li, A. Gray, D. Jiang, X. Mao, Sufficient and necessary conditions of stochastic permanence and extinction for stochastic logistic populations under regime switching, J. Math. Anal. Appl. 376 (2011), 11-28.

[12] M.Liu, et al., Persistence and extinction of a stochastic delay logistic equation under regime switching, Appl. Math. Lett. 26 (2013), 140-144.

[13] M. Liu, K. Wang, Persistence, extinction and global asymptotical stability of a non-autonomous predator-prey model with random perturbation, Appl. Math. Model. 36 (2012), 5344-5353.

[14] X. Mao, C. Yuan, Stochastic Differential Equations with Markovian Switching, Imperial College Press, London, 2006.

[15] X. Mao, G. Marion, E. Renshaw, Environmental Brownian noise suppresses explosions in populations dynamics, Stochastic Process. Appl. 97 (2002), 95-110.

[16] X. Mao, Stochastic Differential Equations and Applications, Horwood Publishing, Chichester, 1997.

[17] T.G. Hallam, Z.E. Ma, Persistence in population models with demographic fluctuations, J. Math. Biol. 24 (1986), $327-339$.

[18] L. Zhang, C. Lu, H. Liu, On a stochastic Lotka-Volterra competitive system with distributed delay and general Levy jumps, Math. Probl. Engineering 2016 (2016), Article ID 3407463.

[19] H. Qiu, W, Deng, Optimal harvesting of a stochastic delay competitive Lotka-Volterra model with Levy jumps, Appl. Math. Comput. 317 (2018), 210-222. 